\title{
Bacillus cereus Fnr binds a [4Fe-4S] cluster and forms a ternary complex with ResD and PlcR
}

Julia Esbelin ${ }^{1,2}$, Yves Jouanneau ${ }^{3,4}$ and Catherine Duport ${ }^{1,2^{*}}$

\begin{abstract}
Background: Bacillus cereus is a facultative anaerobe that causes diarrheal disease in humans. Diarrheal syndrome may result from the secretion of various virulence factors including hemolysin $B L$ and nonhemolytic enterotoxin Nhe. Expression of genes encoding $\mathrm{Hbl}$ and Nhe is regulated by the two redox systems, ResDE and Fnr, and the virulence regulator PlcR. B. cereus Fnr is a member of the Crp/Fnr family of iron-sulfur (Fe-S) proteins. Only its apo-form has so far been studied. A major goal in deciphering the Fnr-dependent regulation of enterotoxin genes is thus to obtain and characterize holoFnr.

Results: Fnr has been subjected to in vitro Fe-S cluster reconstitution under anoxic conditions. UV-visible and EPR spectroscopic analyses together with the chemical estimation of the iron content indicated that Fnr binds one $[4 \mathrm{Fe}-4 \mathrm{~S}]^{2+}$ cluster per monomer. Atmospheric $\mathrm{O}_{2}$ causes disassembly of the Fe-S cluster, which exhibited a half-life of $15 \mathrm{~min}$ in air. Holo- and apoFnr have similar affinities for the nhe and $h b /$ promoter regions, while holoFnr has a higher affinity for fnr promoter region than apoFnr. Both the apo- and holo-form of Fnr interact with ResD and PlcR to form a ternary complex.

Conclusions: Overall, this work shows that incorporation of the $[4 \mathrm{Fe}-4 \mathrm{~S}]^{2+}$ cluster is not required for DNA binding of Fnr to promoter regions of $\mathrm{hbl}$ and nhe enterotoxin genes or for the formation of a ternary complex with ResD and PlcR. This points to some new unusual properties of Fnr that may have physiological relevance in the redox regulation of enterotoxin gene regulation.
\end{abstract}

Keywords: Fnr, Fe-S cluster, anaerobiosis, Bacillus cereus, enterotoxin, DNA binding

\section{Background}

Bacillus cereus is a facultative anaerobic bacterium that can cause two types of food-borne illness in humans. Among these, the diarrheal syndrome may result from the production in the human host's small intestine of various extracellular factors including hemolysin $\mathrm{BL}$ $(\mathrm{Hbl})$ and nonhemolytic enterotoxin Nhe [1,2]. The genes encoding $\mathrm{Hbl}$ and Nhe belong to the PlcR regulon [3]. The ability of B. cereus to produce enterotoxins and grow well in an $\mathrm{O}_{2}$-limited environment such as that prevailing in the human small intestine is controlled by both the two-component system ResDE and the redox

\footnotetext{
*Correspondence: catherine.duport@univ-avignon.fr

'Université d'Avignon et des Pays de Vaucluse, UMR408, Sécurité et Qualité des Produits d'Origine Végétale, F-84000, Avignon, France

2INRA, UMR408, Sécurité et Qualité des Produits d'Origine Végétale, F-84914, Avignon, France

Full list of author information is available at the end of the article
}

regulator Fnr. Unlike ResDE, Fnr is essential for B. cereus growth under anaerobic fermentative conditions and for $h b l$ and nhe expression, irrespective of the oxygenation conditions $[4,5]$. B. cereus $\mathrm{Fnr}$ is a member of the large Fnr/Crp superfamily of transcription factors that bind as homodimers to palindromic sequences of DNA, each subunit binding to one half-site [6]. Like its homologue from Bacillus subtilis, B. cereus Fnr contains a C-terminal extension with four cysteine residues, $C\left(\mathrm{x}_{4}\right)$ $C\left(x_{2}\right) C\left(x_{3}\right) C$. The last three cysteine residues were identified as $[4 \mathrm{Fe}-4 \mathrm{~S}]^{2+}$ cluster ligands in B. subtilis Fnr, the fourth ligand being an aspartate residue [7]. The integrity of this oxygen-labile Fe-S cluster was found to be essential for the DNA binding activity of $B$. subtilis Fnr $[7,8]$. By contrast, B. cereus Fnr appeared active in DNAbinding protein in its apo-form (cluster-free form). This has led to the conclusion that unlike its $B$. subtilis homologue, $B$. cereus Fnr is active in both its apo-form 
and its holo-form (bearing a Fe-S cluster) [9]. However, data evidencing that $B$. cereus Fnr could coordinate a Fe-S cluster under anaerobiosis were lacking.

Here, we show that purified B. cereus apoFnr can bind one $[4 \mathrm{Fe}-4 \mathrm{~S}]^{2+}$ cluster per monomer upon incubation with iron, cysteine and cysteine desulfurase. Reconstituted Fnr (also referred to as holoFnr) showed enhanced DNA binding activity within the $f n r$ promoter, but no activity difference with regard to the $h b l$ and nhe promoters. Both the apo- and holo-form of Fnr interact with ResD and PlcR to form a ternary complex. Our results lend novel insight into the molecular control of enterotoxin gene expression in anaerobically-grown $B$. cereus.

\section{Results}

\section{B. cereus apoFnr binds a labile $[4 \mathrm{Fe}-4 \mathrm{~S}]^{2+}$ cluster}

B. cereus Fnr was expressed as a tag-less polypeptide in aerobically-grown $E$. coli and purified in three steps as described in Methods. The $M_{\mathrm{r}}$ of the purified polypeptide, as estimated by SDS-PAGE under reducing conditions (with DTT) was 25,000, consistent with the theoretical value of 25,640 deduced from the DNA sequence (Additional file 1). The apparent molecular mass of recombinant Fnr, as determined by analytical gel filtration chromatography and by SDS-PAGE under nonreducing conditions (no DTT or $\beta$-mercaptoethanol), was $c a .60,000$, indicating that tag less Fnr occurs mainly as a dimer in solution. As isolated, the Fnr protein was colorless, contains no detectable iron and its UV-visible spectrum did not feature any absorption band other than that at $280 \mathrm{~nm}$ (Figure 1). Thus, we have successfully purified a recombinant tag-less dimeric apo-form of Fnr that is amenable to further investigation in vitro.

The ability of apoFnr to bind an iron-sulfur cluster under anaerobiosis was tested in an enzyme-driven reconstitution system involving the cysteine desulfurase (CsdA) from E. coli (see details in Methods). During anaerobic reconstitution, a brown colour developed resulting from a time-dependent increase of a broad absorbance band around $416 \mathrm{~nm}$, typical for [4Fe-4S] containing proteins (Figure 1). After 90-min reconstitution and subsequent gel filtration, the purified browncolored protein displayed an $A_{416} / A_{280}$ ratio of 0.33 and was found to contain $3.6 \pm 0.1$ moles of iron atoms per mole of monomer. These data are consistent with the reconstitution of one [4Fe-4S] cluster per Fnr monomer $[8,10]$. The reconstituted holoFnr was analysed by EPR spectroscopy, both as isolated and after reduction with sodium dithionite. No signal was detected for the Fnr sample as isolated, but a broad signal with main $g$ values at 2.04, 1.93 was observed upon reduction (Figure 2). These data indicate the presence of a $[4 \mathrm{Fe}-4 \mathrm{~S}]^{2+}$ cluster, which upon one-electron reduction, converted to a paramagnetic $[4 \mathrm{Fe}-4 \mathrm{~S}]^{1+}$ cluster with an electronic spin $\mathrm{S}=1 / 2$. However, the EPR signal differed from that of typical $[4 \mathrm{Fe}-4 \mathrm{~S}]$ proteins in that the resonance lines

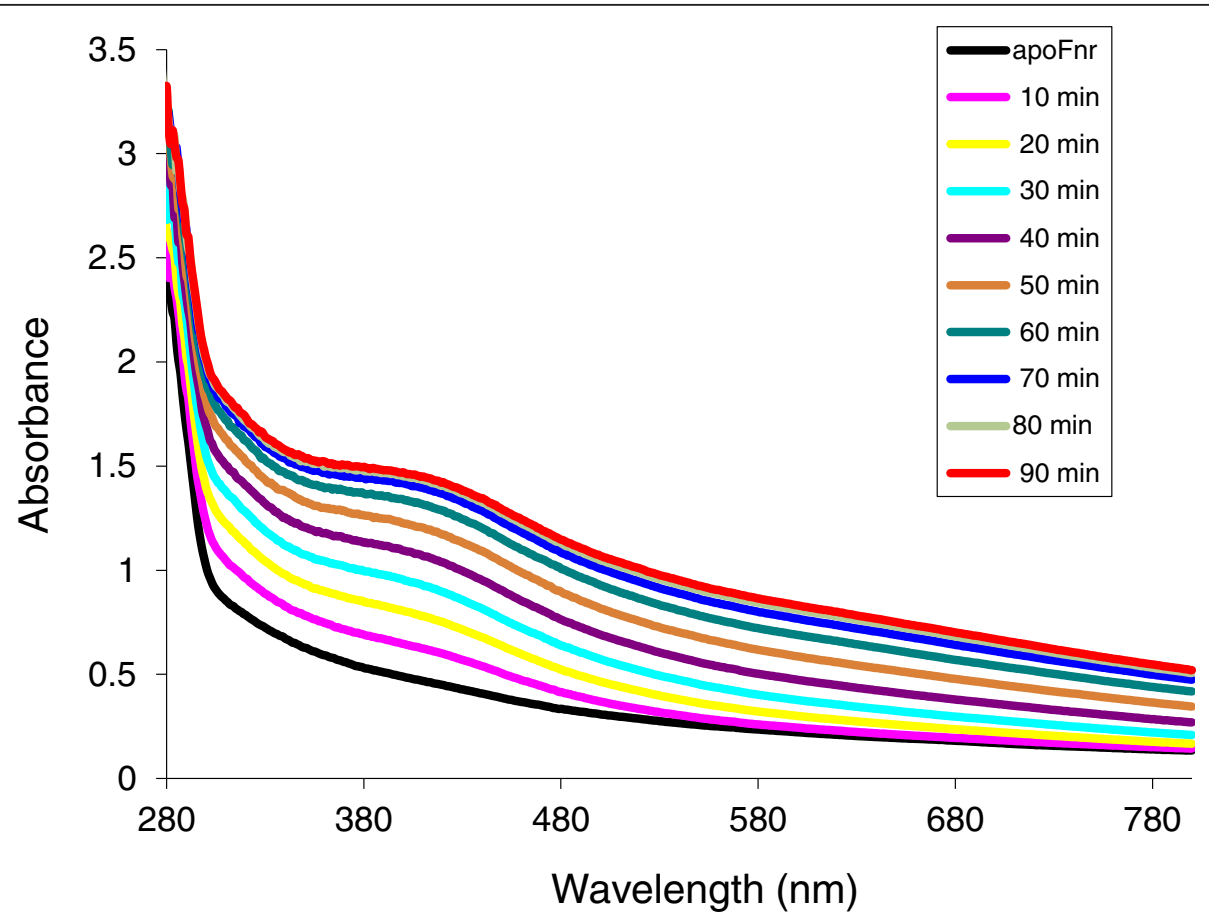

Figure 1 UV-visible spectroscopy of $B$. cereus Fnr Fe-S cluster reconstitution. Reconstitution was carried out inside an anaerobic glove box as described in Methods. Time points at which samples were scanned by a UV-visible spectrophotometer are indicated. 


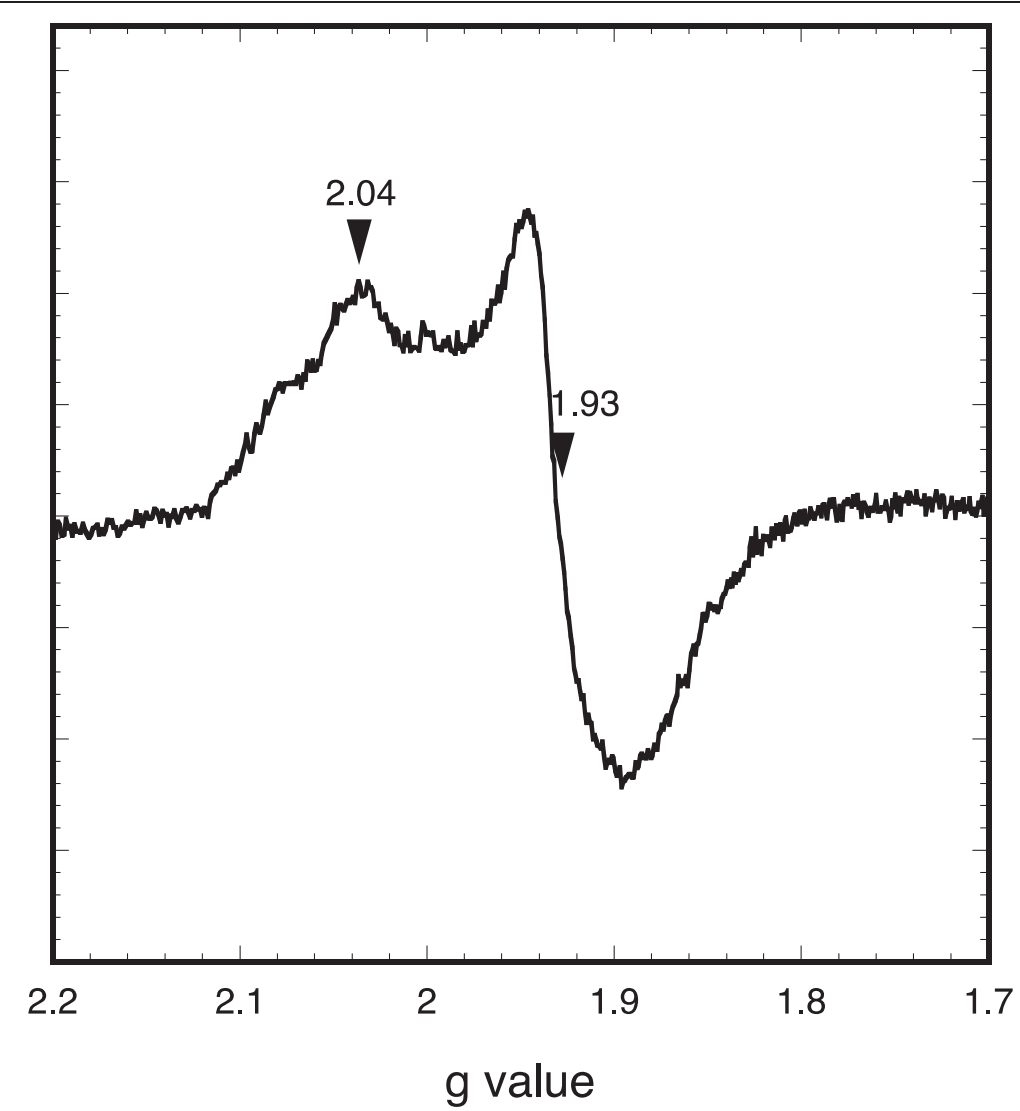

Figure 2 EPR spectrum of $B$. cereus holoFnr after reduction with dithionite. The spectrum was acquired under the following conditions: microwave power $0.1 \mathrm{~mW}$, modulation amplitude $1 \mathrm{mT}$, receiver gain 2.10, temperature $10 \mathrm{~K}$. Relevant $g$ values are indicated.

were relatively broad and showed additional features, especially at high field. As a consequence of this broadening, the $g_{\mathrm{x}}$ component of the tensor was not well resolved. This might reflect some heterogeneity in the vicinity of the cluster, and could be related to the instability of holoFnr upon reduction (see below). In addition, the intensity of the EPR signal was low compared to the protein concentration, although we could not give an accurate estimation of the electronic spin due to the broadening and weakness of the signal. This suggested that the protein was partially reduced, consistent with the observation that dithionite reduction caused a relatively small decrease of the chromophore absorption (data not shown). Attempts to further reduce the protein by using photoreduced 5-deazaflavin were unsuccessful, likely because of the instability of the cluster in the reduced state (data not shown). Taken together, these results suggest that holoFnr contains a redox-responsive [4Fe-4 $\mathrm{S}$ ] cluster, which is unstable upon reduction.

Exposure of reconstituted holoFnr to air resulted in decreased intensity of the $416 \mathrm{~nm}$ absorption band associated with the $[4 \mathrm{Fe}-4 \mathrm{~S}]$ cluster over $60 \mathrm{~min}$
(Figure 3). Based on the absorbance decay at $416 \mathrm{~nm}$, which followed first-order kinetics, the half-life of holoFnr in air was estimated to be $15 \mathrm{~min}$. We conclude that the $[4 \mathrm{Fe}-4 \mathrm{~S}]^{2+}$ cluster of holoFnr was extremely oxygen-labile.

\section{DNA-binding properties of $B$. cereus holoFnr}

The DNA-binding properties of holoFnr were investigated with electrophoretic mobility shift assays (EMSA) under strict anoxic conditions. Figure 4 shows the EMSA results obtained using holo- and apoFnr and the promoter regions of fnr (Figure 4A), nhe (Figure 4B) and $h b l$. Because of its large size $(1,157 \mathrm{bp})$, the promoter region of $h b l$ was divided into two overlapping fragments of $636 \mathrm{bp}$ ( $h b l 1$, Figure 4C) and $610 \mathrm{bp}$ ( $h b l 2$, Figure 4D). The binding specificity was evidenced from the disappearance of the complexes in competition assays using a 50-fold excess of homologous unlabelled promoter regions (data not shown) and by the absence of binding with the negative control (Figure 4E). The results showed that (i) all the complexes formed were stable and did not dissociate during electrophoresis, (ii) the presence of the $[4 \mathrm{Fe}-4 \mathrm{~S}]^{2+}$ cluster increased Fnr-binding 


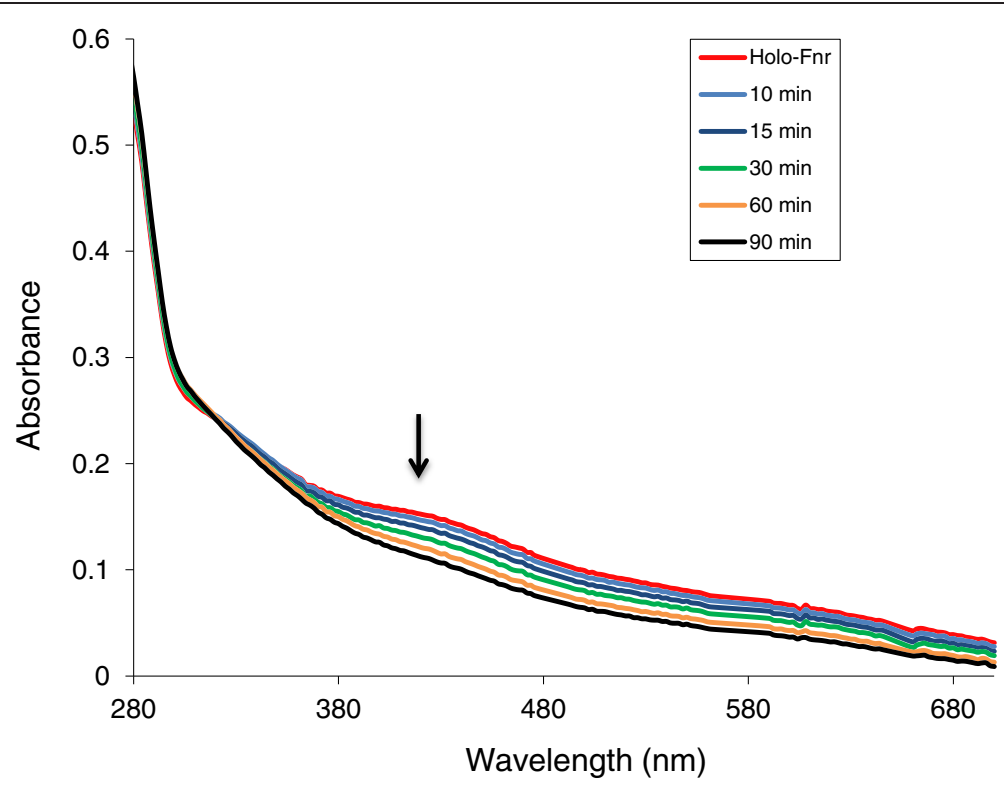

Figure 3 Changes in the ultraviolet/visible spectrum of reconstituted B. cereus Fnr in response to $\mathrm{O}_{2}$. Spectra of $B$. cereus holoFnr [0.56 g/L] were recorded before and $10 \mathrm{~min}, 15 \mathrm{~min}, 30 \mathrm{~min}, 60 \mathrm{~min}$ after exposure to oxygen. Arrow indicates the trend of the spectral changes.

affinity to fnr and nhe promoter regions and did not affect Fnr-binding to $h b l$ promoter regions. Regarding the nhe promoter, the observed difference in apparent binding affinity between the apo- and holo- forms was narrow ( $\leq 1.3$ ). Also, a fairly high level of Fnr (more than $0.6 \mu \mathrm{M}$ ) was needed to form the DNA-Fnr complex. These data suggest that holo- and apoFnr have similar affinities for the nhe promoter.

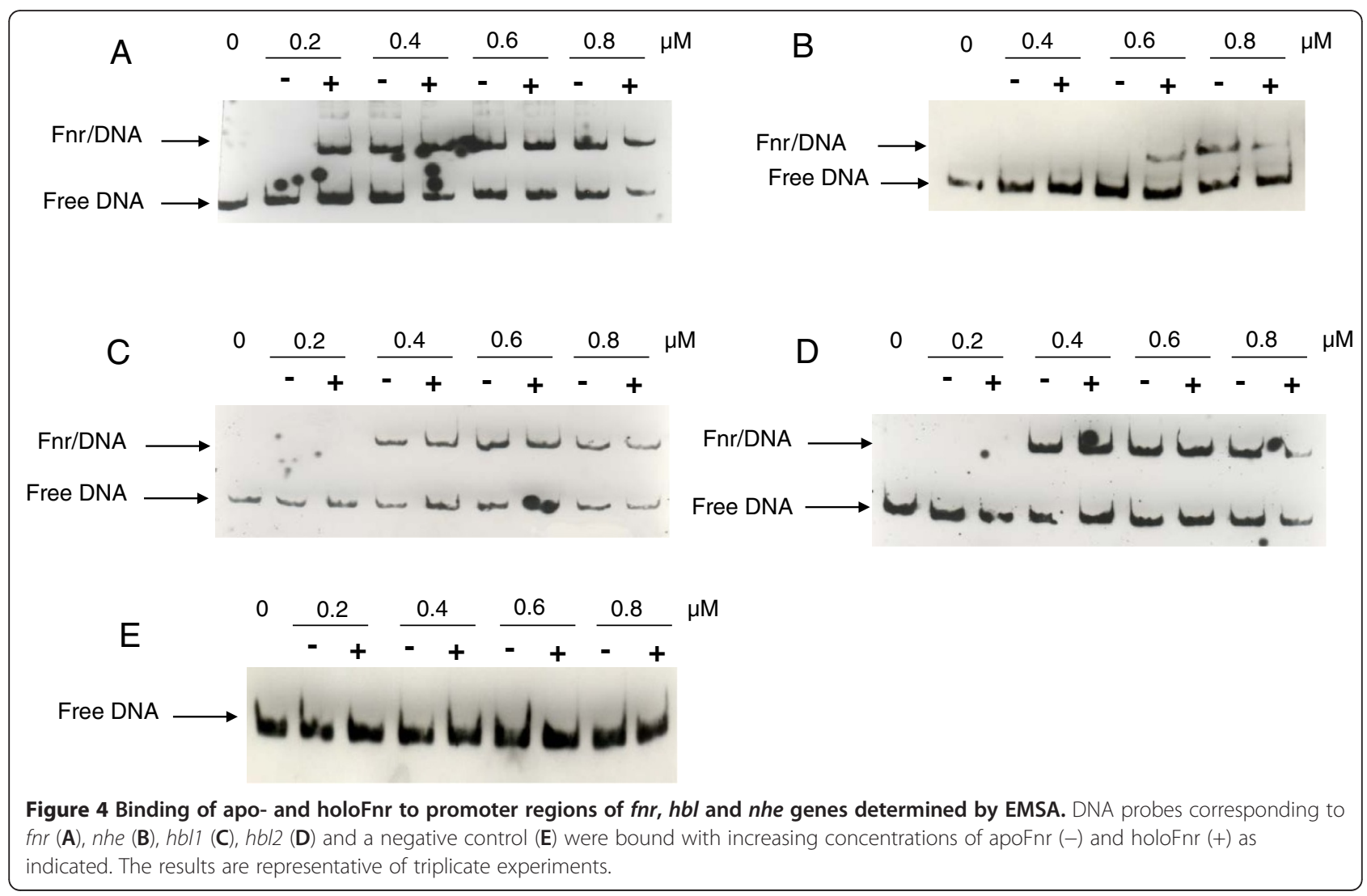




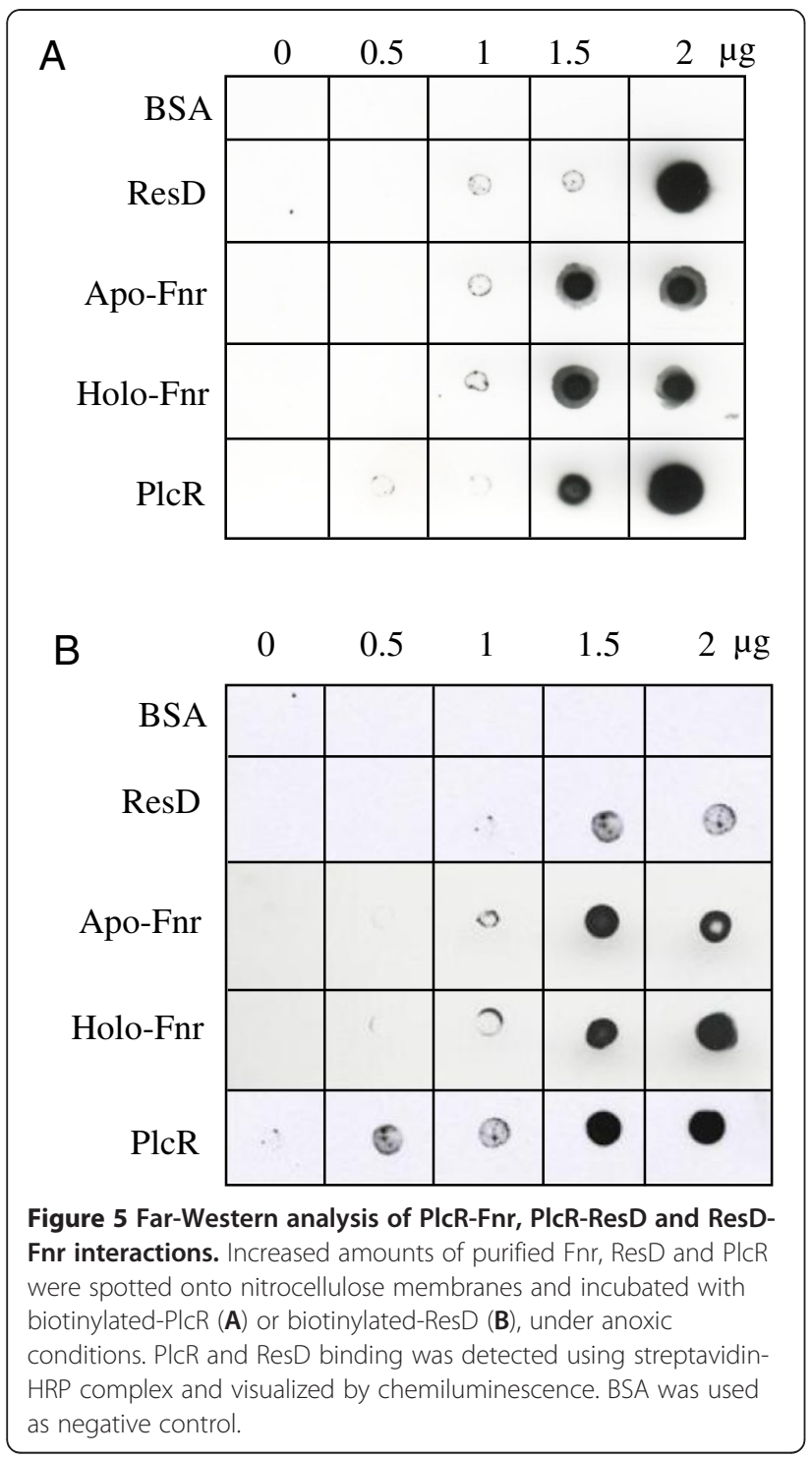

Fnr forms a ternary complex with ResD and PIcR

To determine whether Fnr could interact in vitro with PlcR and ResD, two other regulators of nhe and $h b l$, FarWestern analyses were conducted under anoxic conditions using the apo- and holo- forms of Fnr. Figure 5 shows that (i) BSA (negative control) did not bind to PlcR or ResD, while PlcR and ResD showed self-binding consistent with their capacity to oligomerize [11,12], (ii) both apo- and holoFnr interact with PlcR and ResD and (iii) PlcR interacts with ResD. These pairwise interactions were confirmed by cross-linking experiments using dimethyl suberimidate (Additional file 2).

To determine whether Fnr could interact in vivo with PlcR and ResD, soluble protein extracts were prepared from anaerobically-grown $B$. cereus cells and incubated with anti-Fnr antibodies. Figure 6A shows that antiFnr antibodies could co-precipitate ResD and PlcR

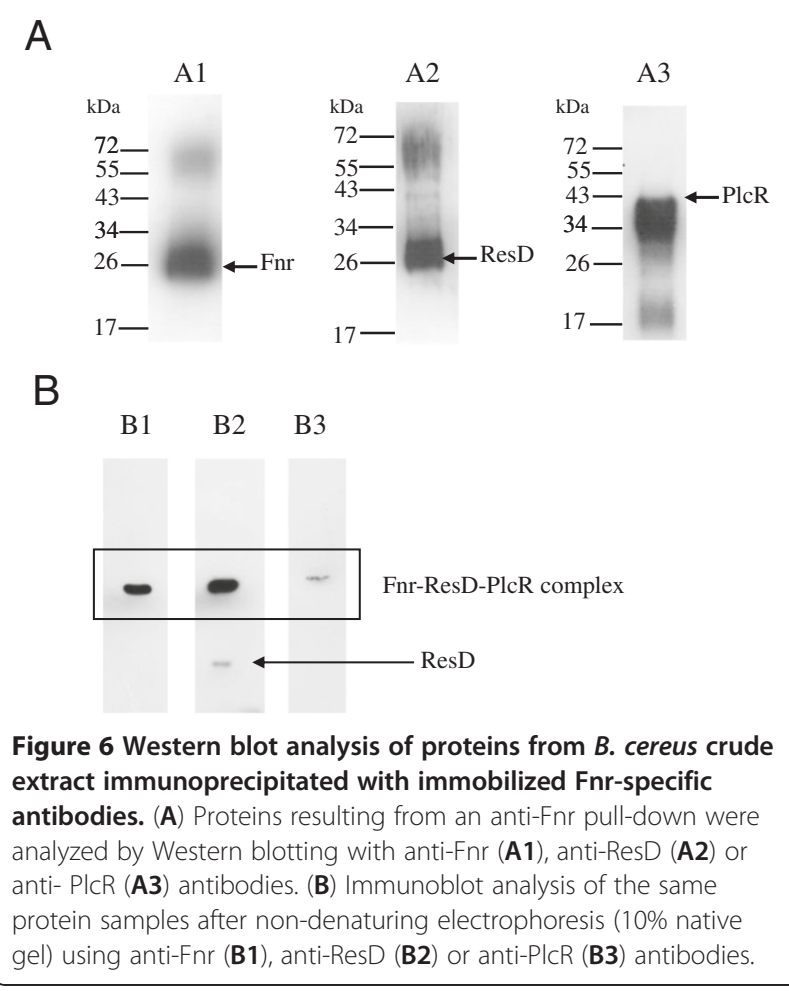

independently. Interestingly, Figure $6 \mathrm{~B}$ shows that antiFnr antibodies co-immunoprecipitated ResD, PlcR and Fnr. These results strongly suggest that Fnr, ResD and PlcR form a ternary complex in vivo.

\section{Discussion}

This work has shown that the Fnr protein of B. cereus is homodimeric and can bind one [4Fe-4 S] iron-sulfur cluster per monomer. Our first challenge was to accurately assemble the Fe-S cluster via an enzymatic system since all our attempts to purify holoFnr under anaerobiosis failed. We demonstrated that CsdA from E. coli was capable of assembling the $B$. cereus Fnr Fe-S cluster. Interestingly, $B$. cereus synthesizes [13] one pyridoxal 5phosphate-containing enzyme (NP_834652) [13] that might be involved in Fe-S cluster biogenesis. When anaerobically reconstituted $B$. cereus Fnr was exposed to $\mathrm{O}_{2}$, we observed a rapid loss of the Fe-S cluster, demonstrating that Fnr functions as an oxygen sensor via its Fe-S cluster. Importantly, the cluster of the reconstituted B. cereus Fnr appeared extremely unstable, judging from its fast destruction on exposure to air. In this respect, the B. subtilis holoFnr, which is the closest homolog of $B$. cereus Fnr [14] displayed greater stability [8]. Sequence comparison of the B. cereus and B. subtilis Fnr revealed a significant variation in the amino acid residues around the three $\mathrm{C}$-terminal cysteine residues (C219- $\left.X_{2}-C_{222}-\mathrm{X}_{4}-\mathrm{C} 227\right)$ that serve as ligands for the cluster (Additional file 3) [7]. These observations imply 
that the occurrence of certain amino acid residues close to the cluster ligands may affect the stability of the $B$. cereus holoFnr, thus providing a possible explanation for its high susceptibility to oxygen damage [15]. As a result, $B$. cereus Fnr might sense subtle changes in the redox status of the cells, a property that would reflect an adaptation of the pathogenic strain to the environment of its human host.

We proposed previously that $B$. cereus apoFnr binds promoter regions of enterotoxins only through the monomer pathway. In other words, we proposed that apoFnr was active as a DNA-binding protein only under its monomeric form [9]. Here we showed that, when produced in a tag-less form, apoFnr is active as a DNA binding protein under its dimeric form. In addition, we showed that dimeric apoFnr-DNA complexes were stable in contrast to what we observed previously [9]. We conclude that (i) in our previous studies, tags fused at the $\mathrm{N}$ terminus and $\mathrm{C}$-terminus of Fnr introduced steric hindrance that affected its oligomeric structure and/or DNA binding activity and (ii) B. cereus apoFnr may bind DNA both through the dimer and the monomer pathway under aerobiosis unlike its homologues of B. subtilis and E. coli $[8]$. There are probably many variables affecting the choice for a monomer or dimer recognition pathway in vivo. Among them, there is the redox state of the cell that may impact directly the ratio of monomeric to dimeric apoFnr since we observed that the addition of reductant (DTT) affected the dimerization state of apoFnr in solution. Finally, the mechanism of apoFnr dependent regulation of enterotoxin is undoubtedly complex, and further extensive experiments are required to examine the role of the monomer and dimer pathways.

The properties of the Fe-S cluster indicate that Fnr is essentially present in the apo- form in aerobically grown B. cereus, and may occur in both apo- and holo- forms in anaerobically-grown bacteria, the ratio between the two forms depending on the redox status of the cells, as detected by the Fnr cluster (Figure 7). The stability of the holo form might also be modulated through interactions with DNA, protein partners and (or) lowmolecular weight thiols [16-18]. Given the higher DNA binding affinity of the holo form compared with the apo form to its own promoter, we assume that higher levels of Fnr (apo + holo) are produced under anaerobiosis than under aerobiosis (Figure 7). In addition, on the basis of these and earlier results, we offer evidence that Fnr can (i) activate the expression of genes encoding the enterotoxin-activators $r e s D$ and $p l c R$ and (ii) associate with PlcR and ResD to form a ternary complex under both anaerobiosis and aerobiosis [4,5,9,11]. By producing higher levels of Fnr [5], anaerobically-grown B. cereus cells might produce higher levels of the tripartite FnrResD-PlcR complex and, as a result, higher levels of $\mathrm{Hbl}$ and Nhe. Hence, the interconversion between apo- and holoFnr may well be a key factor in controlling the regulation of enterotoxin gene expression through the Fnr/ PlcR/ResD complex.

\section{Conclusions}

In conclusion, this work brings further evidence that $B$. cereus Fnr, unlike its counterpart from B. subtilis, is an
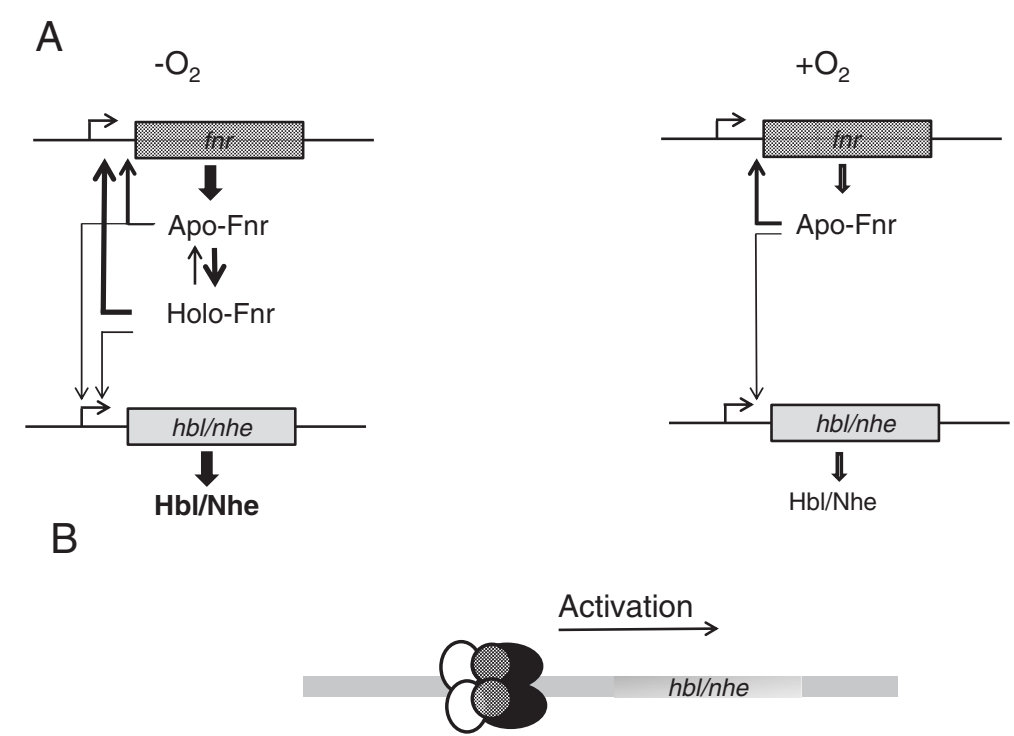

Figure 7 Proposal for the Fnr-dependent regulation of the $\mathbf{h b l}$ and nhe enterotoxin genes in $\mathbf{B}$. cereus. (A) apo- and holoFnr-dependent regulation in either the absence or presence of oxygen. (B), Fnr is thought to be part of a ternary complex involving ResD (black), PlcR (white), Fnr (gray), acting as positive regulator. 
active transcriptional regulator in both its apo- and holo- forms. This property may enable B. cereus to ensure optimal enterotoxin gene expression in response to changes in oxygen tension such as those encountered during infection of the human host.

\section{Methods}

Bacterial strains and growth conditions

Escherichia coli strain TOP10 (Invitrogen) was used as the general cloning host, and strain BL21 CodonPlus (DE3)-RIL (Stratagene) was used to overexpress $f n r$ and resD. E. coli strain BL21 $\lambda \mathrm{DE} 3$, containing the pRep4 plasmid [19] was used to overexpress plcR [12]. E. coli strains were routinely grown in Luria broth at $37^{\circ} \mathrm{C}$.

\section{Recombinant expression of $f n r$, res $D$ and $p / c R$ and protein purifications}

The coding sequence for B. cereus fnr was PCR amplified from F4430/73 genomic DNA using primers PET101F (5'-CACCATGACATTATCTCAAG-3') and PET101R (5'-CTAATCAATGCTACAAACAGAAGC-3'). The amplicon was cloned as a blunt-end PCR product into pET101/D-TOPO (Invitrogen), yielding pET101fur. B. cereus Fnr was produced as a recombinant protein in aerobically grown $E$. coli BL21(pET101fnr). The culture was grown in a 6-litre fermentor until $\mathrm{OD}_{600}$ reached $\sim 1.0$, and protein overproduction was then triggered by $0.2 \mathrm{mM}$ isopropyl-ß-D-thiogalactopyranoside (IPTG). After incubation for $16 \mathrm{~h}$ at $20^{\circ} \mathrm{C}$, cells were harvested by centrifuging at $10,000 \times g$ for $15 \mathrm{~min}$. Fnr was then purified as follows: the bacterial pellet was resuspended in $120 \mathrm{ml}$ of buffer $\mathrm{C}(25 \mathrm{mM}$ Tris- $\mathrm{HCl}$ [pH 8], $1 \mathrm{mM}$ dithiothreitol (DTT)) and incubated with $0.2 \mathrm{mg} \cdot \mathrm{ml}^{-1}$ of lysozyme and $5 \mathrm{mM}$ EDTA for $10 \mathrm{~min}$ at $30^{\circ} \mathrm{C}$. Cells were lysed by ultrasonication for 3 min using a Vibra cell ultrasonifier (Fisher Bioblock Scientific). Cell debris and membrane particles were removed by centrifuging at $43,000 \times g$ for $1 \mathrm{~h}$, and the resulting supernatant was loaded on a $30 \mathrm{ml}$ DEAE-cellulose column (DE52; Whatman) equilibrated with buffer $\mathrm{C}$. The non-retained fraction was adjusted to $\mathrm{pH} 7$ with $1 \mathrm{M} \mathrm{KH}_{2} \mathrm{PO}_{4}$ and then loaded onto a $20 \mathrm{ml}$ hydroxyapatite column (HA Ultrogel; Pall Corporation) equilibrated with buffer D (50 mM KH $\mathrm{PO}_{4}$ [pH 7], $1 \mathrm{mM}$ DTT). The column was developed with a linear gradient from 50 to $200 \mathrm{mM}$ $\mathrm{KH}_{2} \mathrm{PO}_{4}$ at a flow rate of $2 \mathrm{ml} / \mathrm{min}$. Fractions containing recombinant Fnr were pooled and concentrated by ultrafiltration through an Omega disc membrane (30 kDa cutoff, Ø $43 \mathrm{~mm}$, Pall Corporation). A polishing step was then carried out by gel filtration on a column of Superdex SD200 $(1.5 \times 60 \mathrm{~cm}$, Amersham Biosciences $)$ equilibrated with buffer $\mathrm{F}$ (25 mM Tris- $\mathrm{HCl}[\mathrm{pH} 7.5] 1$, $50 \mathrm{mM} \mathrm{NaCl}, 1 \mathrm{mM}$ DTT). The purified protein, >90\% pure by sodium dodecyl sulfate-polyacrylamide gel electrophoresis (SDS-PAGE; Additional file 1), was stored as pellets in liquid nitrogen.

Recombinant expression and purification of resD and $p l c R$ were performed using previously described methods $[11,12]$.

\section{Reconstitution of Fnr holoprotein}

The following procedure was carried out under anoxic conditions $\left(\mathrm{O}_{2}<1 \mathrm{ppm}\right)$ in a glove box maintained at $18^{\circ} \mathrm{C}$ (Jacomex, France). All buffers were degassed under argon and equilibrated for at least $16 \mathrm{~h}$ in the glove box before use. ApoFnr ( $2 \mathrm{~g} / \mathrm{L})$ was incubated with $1 \mu \mathrm{M}$ cysteine desulfurase CsdA from E. coli [20], $1 \mathrm{mML}$ cysteine, and $1 \mathrm{mM} \mathrm{Fe}\left(\mathrm{NH}_{4}\right)_{2}\left(\mathrm{SO}_{4}\right)_{2}$ (Sigma-Aldrich) in the presence of $4 \mathrm{mM}$ DTT in buffer F. Formation of the cluster was monitored by UV-visible spectroscopy using a Uvikon spectrophometer (Kontron) connected through optic fibers to the cuvette holder inside the glove box. The reaction was initiated by adding CsdA, and reached completion after $2 \mathrm{~h}$ (no further increase in the absorption at $416 \mathrm{~nm}$ ). The protein was run through a $10 \mathrm{ml}$ Sephadex G25 column (Amersham Biosciences) equilibrated in buffer $F$ to remove excess reagents, and then concentrated by ultrafiltration using a Nanosep device with a molecular cutoff of $30 \mathrm{kDa}$ (Pall Corporation).

\section{Protein biochemical analyses}

Protein concentrations were determined by either a bicinchoninic acid (BCA) assay (Pierce) or a Biuret method insensitive to thiols [21]. Bovine serum albumin (BSA) was used as a standard. The presence of the Fnr protein in purification fractions was monitored by SDS-PAGE [22], followed by Coomassie blue staining. The iron content of holoFnr was determined spectrophotometrically using a method adapted from Blair and Diehl [23]. Briefly, $50 \mu$ samples of holoFnr $(2.8 \mathrm{~g} / \mathrm{L})$ were incubated at $100^{\circ} \mathrm{C}$ for $15 \mathrm{~min}$ with 30 $\mu \mathrm{L}$ of $6 \mathrm{~N} \mathrm{HCl}$. After dilution to $0.5 \mathrm{ml}$ with $\mathrm{H}_{2} \mathrm{O}$, samples were centrifuged at $12,000 \times g$ for $5 \mathrm{~min}$, and $100 \mu \mathrm{l}$ aliquots of the supernatant fractions were mixed

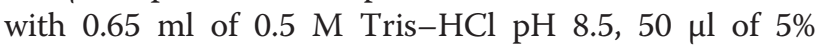
ascorbate and $0.2 \mathrm{ml}$ of $0.1 \%$ bathophenanthroline (Sigma-Aldrich). Mixtures were incubated at room temperature for $1 \mathrm{~h}$, and the absorbance was measured at $536 \mathrm{~nm}\left(\epsilon_{536}=22.14 \mathrm{mM}^{-1} \mathrm{~cm}^{-1}\right)$ and compared with a blank lacking holoFnr.

\section{Spectroscopic characterization of holoFnr}

Samples were prepared in an anaerobic glove box at $18^{\circ} \mathrm{C}$. HoloFnr $(0.1 \mathrm{mM})$ was tentatively reduced with $10 \mu \mathrm{M}$ 5-deazaflavin (a gift from Prof J. Knappe, Heidelberg University, Germany) in the presence of $2.5 \mathrm{mM}$ glycine as electron donor. Photoreduction was 
carried out in a $0.2 \mathrm{~cm}$ light path cuvette by exposing the protein sample to the light of a slide projector for 1 min time periods. Chemical reduction was also applied with an excess of sodium dithionite $(2 \mathrm{mM})$ at $\mathrm{pH}$ 8.5. Progression of the reaction was monitored by recording UV-visible absorption spectra in the 300$700 \mathrm{~nm}$ range.

Samples were transferred into EPR tubes and immediately frozen in liquid nitrogen. EPR spectra were recorded at $10 \mathrm{~K}$ using a Bruker EMX spectrometer equipped with an Oxford Instruments ESR900 liquid helium cryostat.

To assess the sensitivity of holoFnr to oxygen, a fraction of the reconstituted protein was removed from the glove box and exposed to air. Absorbance spectra were recorded at time intervals with an HP8452 diode-array spectrophotometer (Agilent).

\section{Protein-protein interactions}

Far-Western assays and cross-linking reactions were carried out in an anaerobic glove box as described previously [9]. Revelation in Far-Western assays used biotinylated PlcR or biotinylated ResD. The cross-linked products were analyzed by 12\% SDS-PAGE and detected by Western blotting using anti-Fnr and anti-ResD antibodies.

Anaerobic electrophoretic mobility gel shift assay (EMSA) EMSAs were performed in an anaerobic glove box. Fragments containing the promoter regions of $f n r, h b l$, and nhe were PCR-amplified and end-labeled with the following biotinylated primer pairs: FnrFbiot (5'-CGAA CACTTCAGCAGGCATA-3') and FnrR (5'-AATGTC ATACTGTTTGCCAC-3'), Hbl1Fbiot (5'-GGTAAGCAA GTGGGTGAAGC-3') and Hbl1R (5'-AATCGCAAATG CAGAGCACAA-3'), Hbl2Fbiot (5'-TTAACTTAATTCA TATAACTT-3') and Hbl2R (5'-TACGCATTAAAAATT TAAT-3'), NheFbiot (5'-TGTTATTACGACAGTTC CAT-3') and NheR (5'-CTGTAACCAATAACCCTGTG -3 '), respectively. DNA fragment used as negative control was part of sequence BC0007 (NC_004722) and was amplified with the biotinylated primer pairs: F16biot (5'GGTAGTCCACGCCGTAAACG-3') and R16 (5'GAAAACCATGCACCACCTG-3'). The 5'-labeled amplicons were purified using the High Pure PCR Product Purification Kit (Roche). Binding reactions were performed for $30 \mathrm{~min}$ at $37^{\circ} \mathrm{C}$ by incubating biotin-labeled DNA fragments (2 $\mathrm{nM}$ per reaction) with the indicated amount of purified apo- or holoFnr $(0.2,0.4,0.6$ and $0.8 \mu \mathrm{M})$ in $10 \mathrm{mM}$ Tris- $\mathrm{HCl}$ [pH 7.5] buffer containing $50 \mathrm{mM} \mathrm{KCl,} 1 \mathrm{mM}$ DTT, 2.5\% glycerol, $5 \mathrm{mM} \mathrm{MgCl}_{2}$ and $5 \mathrm{mg} / \mathrm{L}$ of poly $(\mathrm{dI}-\mathrm{dC})$. The samples were resolved by electrophoresis on a $6 \%$ non-denaturing polyacrylamide gel [9] and electrotransferred onto Nylon membranes (Amersham Hybond $\mathrm{N}+$ ). Biotin-labeled DNAs were detected using the LightShift Chemiluminescent EMSA Kit (Pierce).

\section{Co-immunoprecipitation}

B. cereus F4430/73 protein lysates were prepared as follows: anaerobically-grown cells were harvested by centrifuging, washed twice with phosphate-buffered saline (PBS; $0.14 \mathrm{M} \mathrm{NaCl}, 2.68 \mathrm{mM} \mathrm{KCl}, 10.14 \mathrm{mM} \mathrm{Na}_{2} \mathrm{HPO}_{4}$, $1.76 \mathrm{mM} \mathrm{KH}_{2} \mathrm{PO}_{4}$ [pH 7.4]), resuspended in lysis buffer (10 mM Tris, 1 mM EDTA, [pH 8]), and mechanically disrupted using a FastPrep instrument (FP120; Bio101, Thermo Electron Corporation). Cell debris were removed by centrifuging $\left(3500 \times g, 10 \mathrm{~min}, 4^{\circ} \mathrm{C}\right)$. The protein lysate was then filtered through a $0.22 \mu \mathrm{m}$ membrane; $100 \mu \mathrm{l}$ of cleared lysate was incubated with $50 \mu \mathrm{l}$ of anti-Fnr protein A-coated Dynabeads prepared by mixing $50 \mu \mathrm{l}$ of polyclonal anti-Fnr [11] with $50 \mu \mathrm{l}$ of protein A Dynabeads (Dynal). The beads were pelleted by centrifuging, washed three times with PBS buffer, and suspended in $20 \mu \mathrm{l}$ of loading buffer. Samples were either directly analyzed by non-denaturing PAGE, or boiled and subjected to $12 \%$ SDS-PAGE. Resolved proteins were transferred to a nitrocellulose membrane (Amersham Bioscience) according to standard procedures (Bio-Rad). Membranes were probed with 1:2,000, 1:1,000 and 1:2,000 dilution of polyclonal rabbit sera raised against Fnr, ResD and PlcR, respectively [9,11,24]. The blotted membranes were developed with 1:2,000 dilution of goat anti-rabbit IgG peroxidase-conjugate (Sigma-Aldrich) and an enhanced chemiluminescence substrate (Immobilon Western, Millipore).

\section{Additional files}

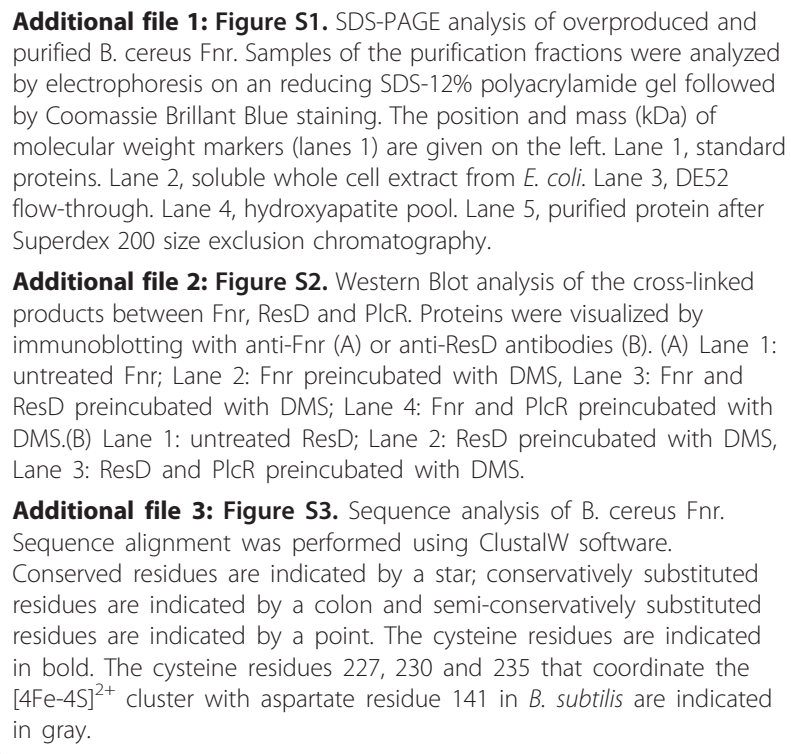

Additional file 2: Figure S2. Western Blot analysis of the cross-linked products between Fnr, ResD and PlcR. Proteins were visualized by immunoblotting with anti-Fnr (A) or anti-ResD antibodies (B). (A) Lane 1: untreated Fnr; Lane 2: Fnr preincubated with DMS, Lane 3: Fnr and ResD preincubated with DMS; Lane 4: Fnr and PlcR preincubated with DMS.(B) Lane 1: untreated ResD; Lane 2: ResD preincubated with DMS, Lane 3: ResD and PICR preincubated with DMS.

Additional file 3: Figure S3. Sequence analysis of B. cereus Fnr. Sequence alignment was performed using ClustalW software. Conserved residues are indicated by a star; conservatively substituted residues are indicated by a colon and semi-conservatively substituted residues are indicated by a point. The cysteine residues are indicated in bold. The cysteine residues 227, 230 and 235 that coordinate the $[4 \mathrm{Fe}-4 \mathrm{~S}]^{2+}$ cluster with aspartate residue 141 in $B$. subtilis are indicated in gray. 


\section{Abbreviations}

Hbl: hemolysin BL; Nhe: non-hemolytic enterotoxin; EMSA: Electrophoretic Mobility Gel Shift Assay.

\section{Competing interests}

The authors declare that they have no competing interests.

\section{Authors' contributions}

JE performed experiments, developed and performed analyses and assays, analyzed the data and contributed to the writing. YJ and CD designed the research, discussed the results and wrote the paper. All authors read and approved the final manuscript.

\section{Acknowledgments}

We thank D. Lereclus for kindly providing plasmids for recombinant expression of plcR and Stephen $H$. Leppla for sending us anti-PICR antibodies. We thank E. Mulliez for the gift of purified CsdA, and S. Ollagnier and E. Mulliez for their help in cluster reconstitution experiments. We also thank $\mathrm{N}$. Duraffourg for recording and comments on the EPR spectra.

\section{Author details}

'Université d'Avignon et des Pays de Vaucluse, UMR408, Sécurité et Qualité des Produits d'Origine Végétale, F-84000, Avignon, France. ${ }^{2}$ INRA, UMR408, Sécurité et Qualité des Produits d'Origine Végétale, F-84914, Avignon, France. ${ }^{3}$ CEA, DSV, iRTSV, Laboratoire de Chimie et Biologie des Métaux, F-38054, Grenoble, France. ${ }^{4}$ UJF-Grenoble 1/CNRS, UMR 5249, F-38041, Grenoble, France.

Received: 6 April 2012 Accepted: 11 June 2012

Published: 25 June 2012

\section{References}

1. Clair G, Roussi S, Armengaud J, Duport C: Expanding the known repertoire of virulence factors produced by Bacillus cereus through early secretome profiling in three redox conditions. Mol Cell Proteomics 2010, 9(7):1486-1498.

2. Stenfors Arnesen LP, Fagerlund A, Granum PE: From soil to gut: Bacillus cereus and its food poisoning toxins. FEMS Microbiol Rev 2008, 32(4):579-606.

3. Gohar M, Faegri K, Perchat S, Ravnum S, Okstad OA, Gominet M, Kolsto AB, Lereclus D: The PIcR virulence regulon of Bacillus cereus. PLoS One 2008, 3(7):e2793.

4. Duport C, Zigha A, Rosenfeld E, Schmitt P: Control of enterotoxin gene expression in Bacillus cereus F4430/73 involves the redox-sensitive ResDE signal transduction system. J Bacteriol 2006, 188(18):6640-6651.

5. Zigha A, Rosenfeld E, Schmitt P, Duport C: The redox regulator Fnr is required for fermentative growth and enterotoxin synthesis in Bacillus cereus F4430/73. J Bacteriol 2007, 189(7):2813-2824.

6. Korner H, Sofia HJ, Zumft WG: Phylogeny of the bacterial superfamily of Crp-Fnr transcription regulators: exploiting the metabolic spectrum by controlling alternative gene programs. FEMS Microbiol Rev 2003, 27(5):559-592.

7. Gruner I, Fradrich C, Bottger LH, Trautwein AX, Jahn D, Hartig E: Aspartate 141 is the fourth ligand of the oxygen-sensing [4Fe-4S] ${ }^{2+}$ cluster of Bacillus subtilis transcriptional regulator Fnr. J Biol Chem 2011, 286(3):2017-2021.

8. Reents $H$, Gruner I, Harmening U, Bottger LH, Layer G, Heathcote $P$, Trautwein AX, Jahn D, Hartig E: Bacillus subtilis Fnr senses oxygen via a [4Fe-4S] cluster coordinated by three cysteine residues without change in the oligomeric state. Mol Microbiol 2006, 60(6):1432-1445.

9. Esbelin J, Jouanneau Y, Armengaud J, Duport C: ApoFnr binds as a monomer to promoters regulating the expression of enterotoxin genes of Bacillus cereus. J Bacterio/ 2008, 190(12):4242-4251.

10. Crack J, Green J, Thomson AJ: Mechanism of oxygen sensing by the bacterial transcription factor fumarate-nitrate reduction (FNR). J Biol Chem 2004, 279(10):9278-9286.

11. Esbelin J, Armengaud J, Zigha A, Duport C: ResDE-dependent regulation of enterotoxin gene expression in Bacillus cereus: evidence for multiple modes of binding for ResD and interaction with Fnr. J Bacterio/ 2009, 191(13):4419-4426
12. Slamti $L$, Lereclus $D$ : A cell-cell signaling peptide activates the PlcR virulence regulon in bacteria of the Bacillus cereus group. EMBO J 2002, 21(17):4550-4559.

13. Clair G, Armengaud J, Duport C: Restricting fermentative potential by proteome remodeling. Mol Cell Proteomics: an adaptive strategy evidenced in Bacillus cereus; 2012. doi:10.1074/mcp.M111.013102.

14. Reents $H$, Munch R, Dammeyer $T$, Jahn D, Hartig E: The Fnr regulon of Bacillus subtilis. J Bacteriol 2006, 188(3):1103-1112.

15. Jervis AJ, Crack JC, White G, Artymiuk PJ, Cheesman MR, Thomson AJ, Le Brun NE, Green J: The $\mathrm{O}_{2}$ sensitivity of the transcription factor FNR is controlled by Ser24 modulating the kinetics of [4Fe-4S] to [2Fe-2S] conversion. Proc Natl Acad Sci U S A 2009, 106(12):4659-4664.

16. Crack JC, den Hengst CD, Jakimowicz P, Subramanian S, Johnson MK, Buttner MJ, Thomson AJ, Le Brun NE: Characterization of [4Fe-4S]containing and cluster-free forms of Streptomyces WhiD. Biochemistry 2009, 48(51):12252-12264.

17. Dey A, Jenney FE Jr: Adams MW, Babini E, Takahashi Y, Fukuyama K, Hodgson KO, Hedman B, Solomon El: Solvent tuning of electrochemical potentials in the active sites of HiPIP versus ferredoxin. Science 2007, 318(5855):1464-1468.

18. Grzyb J, Xu F, Weiner L, Reijerse EJ, Lubitz W, Nanda V, Noy D: De novo design of a non-natural fold for an iron-sulfur protein: alpha-helical coiled-coil with a four-iron four-sulfur cluster binding site in its central core. Biochim Biophys Acta 2010, 1797(3):406-413.

19. Amrein KE, Takacs B, Stieger M, Molnos J, Flint NA, Burn P: Purification and characterization of recombinant human p50csk protein-tyrosine kinase from an Escherichia coli expression system overproducing the bacterial chaperones GroES and GroEL. Proc Natl Acad Sci U S A 1995, 92(4):1048-1052

20. Mihara H, Kurihara T, Yoshimura T, Esaki N: Kinetic and mutational studies of three NifS homologs from Escherichia coli: mechanistic difference between L-cysteine desulfurase and L-selenocysteine lyase reactions. J Biochem 2000, 127(4):559-567.

21. Pelley JW, Garner CW, Little GH: A simple rapid biuret method for the estimation of protein in samples containing thiols. Anal Biochem 1978, 86(1):341-343.

22. Laemmli UK: Cleavage of structural proteins during the assembly of the head of bacteriophage T4. Nature 1970, 227(5259):680-685.

23. Blair D, Diehl H: Bathophenanthroline disulphonic acid and bathocuproine disulphonic acid, water soluble reagents for iron and copper. Talanta 1961, 7:163-174.

24. Pomerantsev AP, Pomerantseva OM, Leppla SH: A spontaneous translational fusion of Bacillus cereus PlcR and PapR activates transcription of PlcR-dependent genes in Bacillus anthracis via binding with a specific palindromic sequence. Infect Immun 2004, 72(10):5814-5823.

doi:10.1186/1471-2180-12-125

Cite this article as: Esbelin et al:: Bacillus cereus Fnr binds a [4Fe-4S] cluster and forms a ternary complex with ResD and PIcR. BMC Microbiology 2012 12:125.

\section{Submit your next manuscript to BioMed Central and take full advantage of:}

- Convenient online submission

- Thorough peer review

- No space constraints or color figure charges

- Immediate publication on acceptance

- Inclusion in PubMed, CAS, Scopus and Google Scholar

- Research which is freely available for redistribution 